\title{
E-Literacia em saúde em jovens adultos: estudo exploratório
}

\section{E-Health literacy in young adults: exploratory study}

\author{
Ana Rita Morais*, Isabel Silva**, Glória Jólluskin** \\ *UFP, ** UFP/Centro de Investigação FPB2S/APASD
}

\begin{abstract}
Resumo
Este estudo pretendeu caracterizar o nível de e-literacia em saúde em jovens adultos portugueses. Participaram 109 indivíduos, com idades entre 18 e 25 anos, que responderam a um questionário sociodemográfico e à Escala de E-literacia em Saúde. Globalmente, os jovens apresentam razoável e-literacia em saúde funcional, níveis medianos de e-literacia crítica, e fraca e-literacia comunicacional. Os resultados revelam não existirem diferenças estatisticamente significativas entre os dois sexos quanto à e-literacia em saúde global, funcional, comunicacional, e crítica, e que a e-literacia crítica em saúde é superior nos participantes que completaram o mestrado do que nos jovens com menor escolaridade. Palavras-chave: literacia, saúde, jovens, internet
\end{abstract}

\begin{abstract}
The present study aimed to characterize the level of ehealth literacy in young Portuguese adults. 109 participants, aged between 18 and 25 , answered to a sociodemographic questionnaire and to the e-Health Literacy Scale. Globally, participants present reasonable functional e-health literacy, median levels of critical ehealth literacy, and poor communicational e-health literacy. The results show that there are no statistically significant differences between the two genders in global, functional, communicational, and critical e-health literacy, and that critical e-health literacy is higher in participants who completed a master degree than in those with lower academic degrees.

Keywords: Literacy, health, young, internet
\end{abstract}

O desenvolvimento da tecnologia e o acesso facilitado à internet tem vindo a mudar a forma como as pessoas assumem o controlo sobre a sua saúde, afetando o modo como estas tomam decisões e fazem escolhas em relação ao seu estilo de vida e saúde, e à prevenção e tratamento de doenças. A internet constitui um importante recurso para acedermos a informações acerca da promoção da saúde e prevenção de doenças (Rempel, Donelle, \& Kothari, 2016), difundido informação sobre saúde tão diversa quanto dados sobre profissionais de saúde ou saúde pública (Weber, Verjee, Rahman, Ameerudeen, \& Al-Baz, 2014).

Neste contexto tecnológico, surge o conceito e-literacia em saúde, entendida como a capacidade de obter, processar, compreender e avaliar informação sobre saúde obtida apartir de meios electrónicos com o objectivo de tomar uma decisão relacionada com a saúde (Norman, \& Skinner, 2006; Norgaard, et al., 2015). A e-literacia em saúde, foi reconhecida como sendo essencial para melhorar os resultados e a qualidade em saúde (Robb, \& Shellenbarger, 2014).

Segundo o relatório 'European Citizens' Digital Health Literacy" (European Comission, 2015) sobre a realidade neste domínio a nível europeu, 6 em cada 10 pessoas utilizam a internet para procurar informação sobre saúde, sendo que essa utilização é feita sobretudo pelos cidadãos entre os 25 e 34 anos e que essa frequência diminui continuamente à medida que a idade aumenta. A maior parte dos cidadãos portugueses que responderam ao inquérito afirmaram que a informação que encontram através da Internet é útil (92\%), que os ajuda a desenvolver os seus conhecimentos sobre saúde (96\%), e que, depois da pesquisa, costumam transmitir as informações sobre saúde que encontraram na internet aos seus familiares e amigos (58\%). Estes dados sugerem os benefícios e potencialidades da e-saúde para a promoção do bem-estar dos usuários, mas existem também algumas potenciais barreiras. Uma parte dos cidadãos portugueses afirma que não é facil perceber a informação que encontram (53\%) e $22 \%$ manifesta mesmo desconhecer como ou onde poderia encontrar informação sobre saúde na internet. Estes resultados são coerentes com o facto de $88 \%$ dos inquiridos terem afirmado que, quando precisam de encontrar informação sobre saúde, tendem a recorrer ao seu médico (European Comission, 2015).

Existem, de facto, alguns fatores que podem condicionar a utilização da internet como recurso para obter informação sobre saúde, alguns dos quais se relacionam com as habilidades e competências dos utilizadores para interagir com as novas tecnologias de forma a atingir os máximos benefícios que a e-literacia pode prover. A investigação revela que $82 \%$ dos cidadãos europeus que afirmam saber utilizar internet para encontrar a informação que precisam para responder às suas questões de saúde costumam utilizar internet diariamente, no entanto estas habilidades relacionadas com a utilização de internet revelam estar relacionadas com as habilitações académicas, sendo que $71 \%$ dos participantes maiores de 20 anos e com estudos ao nível do ensino superior afirmaram utilizar internet todos os dias, descendo esta percentagem aos $25 \%$ no grupo de indivíduos que abandonaram a escola até aos 15 anos (European Comission, 2015). De forma geral, os dados parecem indicar a necessidade geral de promoção da eliteracia em saúde (European Comission, 2015). 
Dados do Instituto Nacional de Estatística [INE] (2016) mostram que a utilização de internet entre os jovens portugueses está amplamente generalizada, sendo que $99 \%$ dos jovens entre 16 e 24 anos e $97 \%$ daqueles entre 25 e 34 anos a utilizam. Estes dados são consistentes com os do relatório europeu sobre literacia em saúde, que mostram que as pessoas mais novas tendem a utilizar diariamente internet (European Comission, 2015). Entre os estudantes é uma prática comum (100\%), assim como entre a população empregada (82\%) (INE, 2016). Assim, parece expectável que os níveis de e-literacia em saúde entre os jovens sejam mais elevados do que na população geral. De facto, Senkowski e Branscum (2015) verificaram que quase todos os jovens acedem diariamente à internet, muitas vezes para procurar informações relacionadas com a saúde. Porém, os dados do relatório europeu sobre literacia em saúde mostram que as pessoas mais novas são o grupo etário que menos satisfeito se sente em relação à informação sobre saúde, por considerar que esta é excessivamente comercial e que não é suficientemente gráfica ou confiável (European Comission, 2015), e a investigação revela que muitos dos jovens ainda manifestam dificuldade em diferenciar entre as informações de elevada e baixa qualidade (Tubaish, \& Habiballah, 2016). Aliás, a preocupação com a qualidade da informação relacionada com a saúde disponibilizada através da internet não é alvo de preocupação apenas pelos jovens, mas por profissionais de saúde, académicos e diversos organismos (Health on the Net Foundation, s.d.; Weber et al. 2014).

Stellefson, Hanik, Chaney, Tennant e Chavarria (2011) constataram que, muito embora os estudantes universitários utilizem frequentemente a internet e se sintam confortáveis com essa ferramenta, os seus níveis de e-literacia em saúde são inferiores ao esperado. Por este motivo, os autores defendem que os jovens deveriam receber formação para desenvolver este tipo de competências, assumindo essa formação ainda maior importante no caso daqueles que se encontram a frequentar em cursos relacionados com a saúde.

A e-literacia em saúde pode constituir-se como uma ferramenta útil nos cuidados de saúde, sendo potenciadora da segurança do doente, assim como dos conhecimentos sobre saúde da população, podendo favorecer a própria tomada de decisão e mudanças relativas a comportamentos de risco, de promoção da saúde e de adesão a tratamentos de doenças (Tubaishat, \& Habiballah, 2016).

Por estes motivos parece importante planificar intervenções de promoção da e-literacia em saúde que permitam não só desenvolver competências de pesquisa, mas também de avaliação da qualidade da informação à qual têm acesso com o objetivo de utilizar de forma mais eficiente essa informação para favorecer a tomada de decisão em saúde. Assim, como primeiro passo, urge perceber o estado da e-literacia em saúde entre os jovens adultos portugueses. Com esse fim, o presente trabalho tem como principal objetivo descrever a e-literacia neste grupo populacional.

\section{Método}

\section{Participantes}

Foi estudada uma amostra de conveniência constituída por 109 participantes, maioritariamente do sexo feminino (73.4\%), com idades compreendidas entre os 18 e os 25 anos; $2.2 \%$ apresentavam uma escolaridade ao nível do $3^{\circ}$ ciclo do ensino básico, $31.2 \%$ do ensino secundário, $53.8 \%$ do $1^{\circ}$ ciclo do ensino superior, e $12.9 \%$ do $2^{\circ}$ ciclo do ensino superior.

\section{Instrumentos}

Foram administrados dois instrumentos de avaliação: um questionário sociodemográfico e a Escala de eLiteracia em Saúde (EeLS).

- Questionário sociodemográfico: desenvolvido especificamente para o presente estudo com o intuito de recolher informação relativa à idade, sexo e habilitações literárias dos participantes.

- EeLS: Esta escala, desenvolvida por Silva e Jólluskin, (versão em estudo), é constituída por 16 itens que procuram avaliar 3 dimensões essenciais da e-literacia em saúde relacionada com o uso de ferramentas eletrónicas (através da internet, nomeadamente páginas web, blogs, chats, redes sociais):

(a) e-Literacia em Saúde Funcional - Subescala constituída por 6 itens, que avaliam a perceção de competência/dificuldade em aceder a informação sobre saúde através dessas ferramentas;

(b) e-Literacia em Saúde Comunicacional - Subescala constituída por 1 item, que avalia a perceção de competência para compreender informação sobre saúde e comunicar a partir de ferramentas eletrónicas;

(c) e-literacia em Saúde Crítica - Subescala constituída por 9 itens, que avaliam a perceção de competência/dificuldade para usar a informação sobre saúde obtida através de ferramentas eletrónicas.

O cálculo do score total e de cada uma das subescalas resulta do somatório das respostas aos itens que as integram, convertido, posteriormente numa escala de percentagem, de forma a facilitar a sua interpretabilidade. Assim, o valor mínimo que é possível obter quer para a escala globalmente considerada, quer para as suas subescalas é 0 e o valor máximo é 100 , sendo que quanto maior é o valor, maior é a perceção de competência de eliteracia em saúde.

No presente estudo, a EeLS apresentou um alfa de Cronbach de .83 , revelador de uma boa fidelidade.

\section{Procedimento}

Foi previamente solicitada a autorização à Comissão de Ética da Universidade Fernando Pessoa para a condução do presente estudo, tendo sido obtido parecer positivo. Procedeu-se, de seguida, ao envio de um convite à participação através de redes sociais e mailing lists institucionais.

Definiram-se como critérios para a seleção dos participantes: (a) serem indivíduos com idade igual ou superior a 18 anos; (b) com língua materna portuguesa; 
(c) e capazes de dar o seu consentimento informado de forma livre.

Todos os questionários foram administrados através de formulário eletrónico, tendo a recolha de dados decorrido entre os meses de fevereiro e abril de 2017. O preenchimento do formulário foi realizado apenas depois dos participantes terem concedido a sua autorização livre e esclarecida, garantindo-se a confidencialidade e anonimato nas respostas.

Os dados recolhidos foram instantaneamente codificados numa base de dados em Excel e, posteriormente, transferidos para o Statistical Package for Social Sciences (SPSS), com o intuito de se proceder à análise estatística dos mesmos.

\section{Resultados}

A análise dos dados revela que, se ao nível da eliteracia em saúde funcional, os participantes não parecem sentir grandes dificuldades, ao nível da eliteracia em saúde crítica parecem avaliar as suas competências como medianas e ao nível da e-literacia em saúde comunicacional revelam não ser frequente comunicarem sobre saúde através de ferramentas eletrónicas (Quadro 1). Verificou-se, ainda, não existirem diferenças estatisticamente significativas entre os dois sexos quanto à e-literacia em saúde globalmente considerada $(U=489.50 ; p>.05)$, nem quando aos domínios específicos avaliados - e-literacia em saúde funcional $\quad(U=756.00 ; \quad p>.05), \quad$ comunicacional $(U=983.50 ; p>.05)$ e crítica $(U=720.50 ; p>.05)$ (Quadro 1).

Quadro 1.

Análise descritiva de competências de e-literacia em saúde e comparação entre participantes dos dois sexos

\begin{tabular}{lcc}
\hline & Sexo & Sexo \\
e-Literacia em Saúde & feminino & masculino \\
& $D P$ & $M$ \\
\hline Total & 61.42 & $6 P$ \\
e-Literacia em Saúde & 11.91 & 13.32 \\
Funcional & 75.00 & 71.74 \\
e-Literacia em Saúde & 15.34 & 15.44 \\
Comunicacional & 21.00 & 32.00 \\
e-Literacia em Saúde Crítica & 23.62 & 26.54 \\
\hline
\end{tabular}

Quanto à escolaridade, observou-se não existirem diferenças estatisticamente significativas entre participantes com distintos níveis de escolaridade em relação à e-literacia em saúde globalmente considerada $(F=2.213 ; p>.05)$, à e-literacia em saúde funcional $(F=.957 ; p>.05)$ e à e-literacia em saúde comunicacional $((F=.750 ; p>.05)$. Porém, relativamente à e-literacia em saúde crítica $(F=2.94 ; p<.05)$, a análise dos dados aponta no sentido de que os participantes que apresentam uma escolaridade ao nível do $2^{\circ}$ ciclo de estudos no ensino superior sejam aqueles que revelam possuir perceção de maior competência/menor dificuldade neste domínio, ainda essas competências que não deixem de ser por eles avaliadas como medianas (Quadro 2).

Quadro 2.

Diferenças entre participantes com diferentes níveis de escolaridade quanto à sua perceção de competência no domínio da e-Literacia em Saúde Crítica

\begin{tabular}{lcc}
\hline Nível de escolaridade & \multicolumn{2}{c}{ e-Literacia em Saúde Crítica } \\
\hline & $M$ & $D P$ \\
$3^{\text {o }}$ ciclo do ensino básico & 57.40 & 4.24 \\
Ensino secundário & 49.56 & 12.82 \\
$1^{\circ}$ ciclo do ensino superior & 50.62 & 16.88 \\
$2^{\circ}$ ciclo do ensino superior & 61.88 & 14.22 \\
\hline
\end{tabular}

\section{Discussão}

Globalmente, os jovens apresentam níveis razoáveis de e-Literacia em Saúde Funcional e níveis medianos de eLiteracia em Saúde Crítica, porém destaca-se o facto de apresentarem uma e-Literacia em Saúde Comunicacional fraca. Os resultados revelam não existirem diferenças estatisticamente significativas entre os dois sexos relativamente à e-Literacia Geral, e à e-Literacia Funcional, Comunicacional e Crítica. Quanto à escolaridade, observou-se não existirem diferenças estatisticamente significativas entre participantes com distintos níveis de escolaridade em relação à e-Literacia Geral, e-Literacia Funcional e e-Literacia Comunicacional. Porém, relativamente à e-Literacia Crítica, a análise dos dados aponta no sentido de que os participantes que apresentam uma escolaridade ao nível do $2^{\circ}$ ciclo de estudos no ensino superior sejam aqueles que revelam possuir maior e-Literacia Crítica em saúde, ainda que, mesmo assim, considerem essa sua competência como mediana. Tal parece ser concordante com os dados de outros estudos, que já alertavam para algumas dificuldades relatadas pelos jovens, nomeadamente para diferenciar entre as informações relacionadas com a saúde de elevada e reduzida qualidade (Tubaish, \& Habiballah, 2016).

Os resultados sugerem, assim, que urge o planeamento de programas de promoção da literacia em saúde, que deverão prestar particular atenção à e-literacia comunicacional e à e-literacia crítica, isto é, ao desenvolvimento de competências relacionadas com a compreensão de informação sobre saúde e de comunicação neste domínio através de ferramentas eletrónicas, bem como à promoção da capacidade para utilizar de forma crítica e reflexiva informação sobre saúde disponibilizada por meios eletrónicos, capacitando os jovens para avaliarem a qualidade dessa informação e para identificarem fontes de informação eletrónicas confiáveis, potenciando a sua proatividade em saúde e a tomada de decisão em saúde a partir de recursos disponíveis na internet.

\section{Referências}

European Commission (2015). Flash Eurobarometer 404 (European Citizens' Digital Health Literacy). TNS Political \& Social [producer]. GESIS Data Archive, 
Cologne. ZA5948 Data file Version 1.0.0, doi:10.4232/1.12194 Retrieved from: http://ec.europa.eu/commfrontoffice/publicopinion/fla sh/fl_404_en.pdf

Health on the Net Foundation (s.d.). The commitment to reliable health and medical information on the internet. $\quad$ Retrieved from http://www.hon.ch/HONcode/Pro/Visitor/visitor.html

Instituto Nacional de Estatística (2016). Inquérito à utilização de tecnologías da informação e da comunidade pelas familias. Retrieved from: https://www.ine.pt/xportal/xmain?xpid=INE\&xpgid=i ne_destaques\&DESTAQUESdest_boui $=250254698 \&$ DESTAQUESmodo $=2$

Norman, C. D., \& Skinner, H. A. (2006). eHEALS: The eHealth literacy scale. Journal of Medical Internet Research, 8(4), e27. doi: 10.2196/jmir.8.4.e27

Norgaard, O., Furstrand, D., Klokker, L., Karnoe, A., Batterham, R., Kayser, L., \& Osborne, R. (2015). The e-health literacy framework: A conceptual framework for characterizing e-health users and their interaction with e-health systems. Knowledge Management \& ELearning, 7(4), 522-540.

Rempel, E., Donelle, L., \& Kothari, A. (2016). Exploring the health literacy of first nations Young adult women. Journal of Communication in Healthcare, 1(9), 11-21. http://dx.doi.org/10.1080/17538068.2015.1122873

Robb, M., \& Shellenbarger, T. (2014). Influential factors and perceptions of eHealth literacy among undergraduate college students. Online Journal of Nursing Informatics, 18 (3). Retrieved from http://www.himss.org/influential-factors-andperceptions-ehealth-literacy-among-undergraduatecollege-students

Senkowski, V., \& Branscum, P. (2015). How college students search the internet for weight control and weight management information an observational study. American Journal of Health Education, 46, 231240.

http://dx.doi.org/10.1080/19325037.2015.1044139

Stellefson, M. L., Hanik, B., Chaney, B., \& Chavarria, R. (2011). eHealth literacy among college students: a systematic review with implications for eHealth education. Journal of Medical Internet Research 13(4):e10. doi: 10.2196/jmir.1703

Tubaishat, A., \& Habiballah, L. (2016). Ehealth literacy among undergraduate nursing students. Nurse Education Today, 42, 47-52. https://doi.org/10.1016/j.nedt.2016.04.003

Weber, A.S., Verjee, M., Rahman, Z.H., Ameerudeen, F., \& Al-Baz, N. (2014). Typology and credibility of Internet health websites originating from Gulf Cooperation Council countries. Eastern Mediterranean Health Journal, 20(12), 804-811. 